\title{
The Rate Distortion Region for the Multiple Description Problem ${ }^{1}$
}

\author{
Michael Fleming \\ Dept. of Electrical Eng. (136-93) \\ California Institute of Technology \\ Pasadena, CA 91125, USA
}

\author{
Michelle Effros \\ Dept. of Electrical Eng. (136-93) \\ California Institute of Technology \\ Pasadena, CA 91125, USA
}

\begin{abstract}
We derive the rate-distortion region for the two-channel multiple description problem on stationary discrete ergodic and nonergodic sources with alphabets admitting an ergodic decomposition. The results do not provide a single-letter representation for the rate-distortion region on i.i.d. sources.
\end{abstract}

\section{INTRODUCTION}

In multiple description (MD) source coding with two channels, a source is described at two different rates, and each description is sent over a separate channel to the receiver. Each channel has some probability of breaking down, in which case all of the data sent on that channel is lost. If only channel $i$ is working, the receiver makes reproduction $\hat{X}_{i}$ with average distortion $D_{i}$ using the rate- $R_{i}$ description sent on channel $i$. When both channels work, the receiver makes reproduction $\hat{X}_{12}$ with average distortion $D_{12}$ using the description provided by combining the information on both channels with an additional rate- $R_{12}$ description. The descriptions of both $\hat{X}_{1}$ and $\hat{X}_{2}$ are available when decoding $\hat{X}_{12}$; the additional rate $R_{12}$ spent on $\hat{X}_{12}$ can be treated as refinement and split arbitrarily between the two channels.

Other authors have found upper and lower bounds on the achievable rate distortion region (the set of achievable vectors $\left.(\mathbf{R}, \mathbf{D})=\left(R_{1}, R_{2}, R_{12}, D_{1}, D_{2}, D_{12}\right)\right)$ for the two-channel multiple description of an i.i.d. source. The bounds do not match for all sources. We present a new achievability theorem and matching converse giving the achievable rate-distortion region for both ergodic and nonergodic sources. On i.i.d. sources, our converse is similar to that of [1], and our achievability result uses an existing achievability result from [2]. We use a Lagrangian approach and closely parallel [3].

Both the converse and achievability proofs make use of distributions with a property that we call $n$-block conditional independence, defined in the following section. The use of distributions with this property arises from the observation that the bounds of [1] and [2] match when $\hat{X}_{1}$ and $\hat{X}_{2}$ are conditionally independent given $X$. While such conditional independence is not observed on a symbol-by-symbol basis, it arises naturally when using $n$-dimensional codes since $\hat{X}_{1}^{n}, \hat{X}_{2}^{n}$ and $\hat{X}_{12}^{n}$ are all uniquely determined by $X^{n}$.

\section{N-Block Conditional InDEPENDENCE}

Let the elements of a one-sided infinite sequence $Y$ be denoted $Y_{1}, Y_{2}, \ldots$ We divide these elements into $n$ blocks as $\mathbf{Y}(i)=Y_{(i-1) n+1}^{\text {in }}$. We say that distribution $\mathbf{q}\left(\hat{\mathbf{x}}_{12}, \hat{\mathbf{x}}_{1}, \hat{\mathbf{x}}_{2} \mid \mathbf{x}\right)$ has $n$-block conditional independence if $\mathbf{q}\left(\hat{\mathbf{x}}_{12}, \hat{\mathbf{x}}_{1}, \hat{\mathbf{x}}_{2} \mid \mathbf{x}\right)=\prod_{k=1}^{\infty} q_{c}^{n}\left(\hat{\mathbf{x}}_{12}(k), \hat{\mathbf{x}}_{1}(k), \hat{\mathbf{x}}_{2}(k) \mid \mathbf{x}(k)\right)$, and $q_{c}^{n}\left(\hat{x}_{1}^{n}, \hat{x}_{2}^{n} \mid x^{n}\right)=q_{c}^{n}\left(\hat{x}_{1}^{n} \mid x^{n}\right) q_{c}^{n}\left(\hat{x}_{2}^{n} \mid x^{n}\right)$. Define $\Gamma(n)$ to be the

${ }^{1}$ This work was supported by a Pickering Fellowship, NSF grant CCR-9909026, Caltech's Lee Center for Advanced Networking, an F.W.W. Rhodes Memorial Scholarship, and a Redshaw Award. set of all distributions $\mathbf{q}\left(\hat{\mathbf{x}}_{12}, \hat{\mathbf{x}}_{1}, \hat{\mathbf{x}}_{2} \mid \mathbf{x}\right)$ with $n$-block conditional independence for a particular $n$, and let $\Gamma=\bigcup_{n=1}^{\infty} \Gamma(n)$.

\section{RESULTS}

Let $A$ be a discrete source alphabet, and define $A^{\infty}$ to be the set of one-sided sequences from $A$. Let $p$ be a stationary source with alphabet $A^{\infty}$ and marginal $p^{n}$ on $A^{n}$. Let $\hat{A}$ be a discrete reproduction alphabet, and let $\rho: A \times \hat{A} \rightarrow[0, \infty)$ be a nonnegative distortion measure. We assume that there exists a reference letter $y^{*} \in \hat{A}$ such that $E_{p} \rho(x, y)=d^{*}<\infty$. Define $\rho\left(x^{n}, y^{n}\right)=\sum_{i=1}^{n} \rho\left(x_{i}, y_{i}\right)$.

We denote an MD quantizer of blocklength $n$ by $Q^{n}=$ $\left(Q_{1}^{n}, Q_{2}^{n}, Q_{12}^{n}\right)$. For each $S, Q_{S}^{n}$ maps $A^{n}$ onto some finite or countable set of codewords $\mathcal{C}_{S}^{n}$ from $\hat{A}^{n}$. We assume that the description of $\hat{X}_{12}^{n}$ made by $Q_{12}^{n}$ is a refinement of the descriptions $\hat{X}_{1}^{n}$ and $\hat{X}_{2}^{n}$ made by $Q_{1}^{n}$ and $Q_{2}^{n}$ respectively, since these individual descriptions are available to the decoder when decoding $\hat{X}_{12}^{n}$. The codeword descriptions are assumed to be uniquely decodable.

The set $\mathcal{R}(p)$ of asymptotically achievable rate-distortion vectors $(\mathbf{R}, \mathbf{D})$ is, by a timesharing argument, a convex set, and can be entirely characterized by its support functional $j(\alpha, \beta, \mathbf{p})=\inf _{(\mathbf{r}, \mathrm{d}) \in \mathcal{R}(p)} \sum_{S \in \mathcal{M}}\left(\alpha_{S} d_{S}+\beta_{S} r_{S}\right)$, where $\mathcal{M}=$ $\{\{1\},\{2\},\{12\}\}$.

The weighted rate-distortion function is defined as

$$
J(\alpha, \beta, \mathbf{p})=\inf _{n} J_{n}(\alpha, \beta, \mathbf{p}),
$$

where

$$
\begin{aligned}
& J_{n}(\alpha, \beta, \mathbf{p})=\inf _{\mathbf{q} \in \Gamma(n)} \frac{1}{n}\left[\sum_{S \in \mathcal{M}} \alpha_{S} E_{p^{n} q_{c}^{n}} \rho\left(X^{n}, \hat{X}_{S}^{n}\right)\right. \\
& \left.\quad+\sum_{i=1}^{2} \beta_{i} I_{p^{n} q_{c}^{n}}\left(X^{n} ; \hat{X}_{i}^{n}\right)+\beta_{12} I_{p^{n} q_{c}^{n}}\left(X^{n} ; \hat{X}_{12}^{n} \mid \hat{X}_{1}^{n}, \hat{X}_{2}^{n}\right)\right] .
\end{aligned}
$$

Stationary Ergodic Sources: When $\mathbf{p}$ is stationary and ergodic, the following result holds.

Theorem 1: $j(\alpha, \beta, \mathbf{p})=J(\alpha, \beta, \mathbf{p})$.

Stationary Nonergodic Sources: When $\mathbf{p}$ is stationary and nonergodic, but an ergodic decomposition $\left\{\mathbf{p}_{\mathbf{x}}: \mathbf{x} \in A^{\infty}\right\}$ of $\mathbf{p}$ exists, then the following results hold.

Theorem 2: $J(\alpha, \beta, \mathbf{p})=\int J\left(\alpha, \beta, \mathbf{p}_{\mathbf{x}}\right) d p(\mathbf{x})$.

Theorem 3: $j(\alpha, \beta, \mathbf{p})=J(\alpha, \beta, \mathbf{p})$.

\section{REFERENCES}

[1] S. Sher and M. Feder, "A converse theorem for the multiple description problem," Eighteenth Convention of Electrical and Electronics Engineers in Israel, pp. 1.1.4/1-4, Mar. 1995.

[2] A. El Gamal and T.M. Cover, "Achievable rates for multiple descriptions," IEEE Trans. Info. Theory, vol. 28, pp. 851-857, Nov. 1982.

[3] M. Effros, "Distortion-rate bounds for fixed- and variable-rate multi-resolution source codes," IEEE Trans. Info. Theory, vol. 45, pp. 1887-1910, Sep. 1999. 